\title{
Multi-Scale Blobs for Saliency Detection in Satellite Images
}

\author{
Yanan Zhou ${ }^{1,2,3} \cdot$ Jiancheng $\mathrm{Luo}^{2} \cdot$ Xiaodong $\mathrm{Hu}^{2,3} \cdot$ Zhanfeng Shen $^{2}$
}

Received: 5 November 2013 / Accepted: 19 June 2015 /Published online: 3 December 2015

(C) Indian Society of Remote Sensing 2015

\begin{abstract}
Saliency can be modeled as spatially localized and contrasted structures with higher blob density and higher blob evenness across scales in images. And it is likely to contain regions and objects of interest. So saliency detection is desired before further image processing and analysis. This paper presented an automatic and effective method for saliency detection in satellite images, based on multi-scale blob information. Firstly, multi-scale blob information were extracted from input images, to produce a blob map. Then, in the blob map, multi-level distance transform spread the blob information to the entire image, to generate a saliency map. Finally the saliency map was segmented to detect salient regions and to locate object centers. The experimental results illustrated its accuracy and stability for detecting salient regions (such as residential areas, parking lots and airplane docks) and for locating object centers in various satellite images.
\end{abstract}

Keywords Multi-scale blob · Multi-level distance transform · Salient region $\cdot$ Object center $\cdot$ Satellite image

Yanan Zhou

zhounanq@gmail.com

1 Institute of Geographic Sciences and Natural Resources Research, Chinese Academy Sciences, Beijing 100101, China

2 Institute of Remote Sensing and Digital Earth, Chinese Academy of Sciences, Beijing 100101, China

3 College of Geophysics, Chengdu University of Technology, Chengdu, Sichuan 610059, China

\section{Introduction}

With the large amount of satellite images available, it has become very difficult for image analysis and understanding by humans or machines (Xu et al. 2007; Hu et al. 2013). The huge size of images also imposes heavy computational and time constraints. On the other hand, many applications, such as region/ object detection, are only focusing on some salient regions or objects in images (Itti et al. 1998; Xu et al. 2007). It is desired to extract regions and objects of interest before further image processing and analysis (Cui et al. 2014; Zhang et al. 2015).

Saliency detection is an important method to extract the regions and objects of interest. It is designed to select a subset of the available sensory information before further processing and to extract the most relevant features, most likely to reduce the time and complexity of scene analysis (Jäger and Hellwich 2005; Li and Pan 2011). Due to such superiority, saliency detection has been adopted in widely applications, including image segmentation, land cover and land use classification ( $\mathrm{Li}$ and Itti 2011), and distinctive objects detection (Chalmond et al. 2006; Wang et al. 2013).

Without a specific task, feature-driven informative patch extraction is one of the most effective saliency detection methods (Shapiro and Stockman 2001; Hu et al. 2013; Chen et al. 2014). And it is widely used for extraction of interesting regions and object recognition in natural images (Rosin 2009; Klein and Frintrop 2011) and satellite images (Li and Itti 2011; Chen et al. 2014; Zhang et al. 2015). Essentially, saliency at each location is determined by its dissimilarity from its neighborhood in terms of density, orientation, variance, junction, and edge density (Rosin 2009; Hu et al. 2013). Each basic feature is computed, summed and further combined to generate the saliency map. Xu et al. (2007) introduced a saliency detection method based on Itti's saliency model (Itti et al. 1998) to extract salient objects form panchromatic 


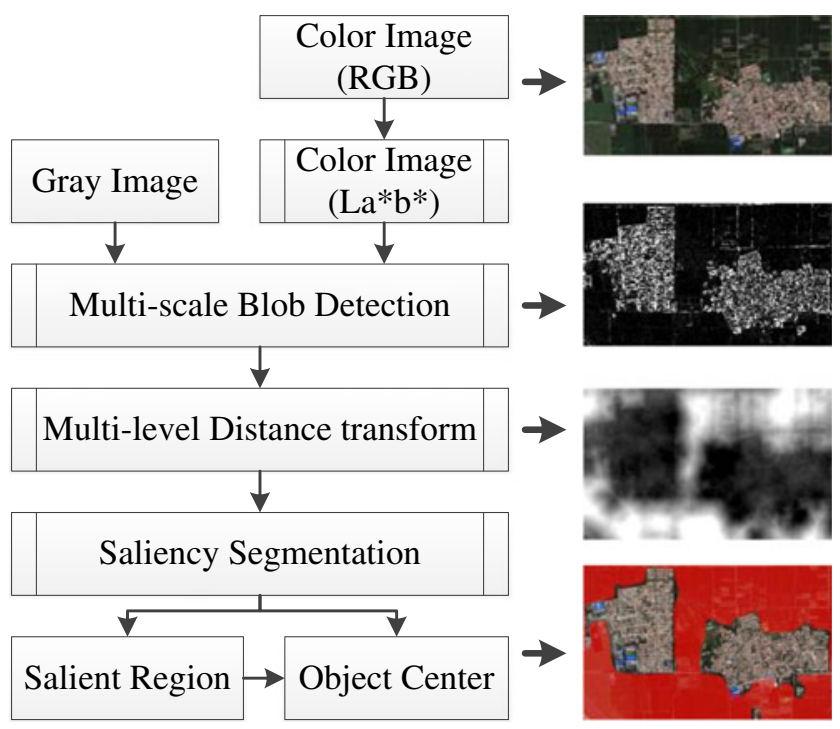

Fig. 1 Workflow of saliency detection

satellite images. Harel et al. (2006) created feature maps using Itti's method but performed their normalization using the graph-based visual saliency approach. The results show a remarkable consistency with salient objects. Cui et al. (2014) proposed a novel top-down saliency detection method using local adaptive regression kernels, measuring the likeness of image patches to a given single template image. However, it has been noted that those approaches require many design parameters such as the number, types and sizes of the filters, as well as weights, normalization schemes.

Differently from the above methods, Rosin (2009) proposed a simple scheme to produce saliency maps based on edge density, and obtained promising results in natural images. Hu et al. (2013) further incorporated local edge distributions for detection of salient structure textures and objects in satellite images.

Inspired by Rosin's method and Hu's method, we proposed an automatic and effective approach for saliency detection based on multi-scale blobs in satellite images. In this paper, a blob is defined as a very small region of connected pixels, more than edges and key points. And the blob in images reveals visual uniqueness in discontinuity, contrast, evenness, and structure pattern (Lowe 2004). Compared with the existing works, the contributions of our work were threefold. First, multi-scale blobs resulted from a new method were taken as the main information source to calculate saliency maps, rather than image edges (Rosin 2009) at a special scale (Hu et al. 2013). Secondly we incorporated the blob distribution evenness in scale space to enhance region saliency. Thirdly we employed a stable and precise statistical method to locating object centers in local saliency regions. Actual satellite images covering various ground features were used for detecting salient regions and locating object centers, compared with Rosin's method and Hu's method.

\section{Workflow of Saliency Detection}

In this paper, we presented an automatic approach to detect salient regions and objects based on multi-scale blobs in satellite images. Our method is based on the remark that image saliency should be detected from multiple scales, since bottom features (color, edge, blob, texture, etc.) at a special scale can only identify salient objects of the corresponding scale (Shapiro and Stockman 2001; Chalmond et al. 2006; Zhang et al. 2015).

Color information in high-resolution satellite images is not as rich as that in natural images (Chen et al. 2014). And panchromatic images are more available than multispectral images. So for color satellite images, we first transformed them from $R G B$ color space into $L a^{*} b^{*}$ color space with $L$ for lightness channel, and $a$ and $b$ for the color-opponent channel. Then the [0255] normalized $L$ channel or gray images were taken as input data for the workflow. So the proposed method was able to handle color images as well as gray images. Figure 1 shows the workflow of saliency detection.

Gaussian smoothing and bilateral filtering algorithm were firstly used to detect multi-scale blobs and generate a blob map from the input image. Then in the blob map, a multi-level distance transform spread blob information to the entire image, producing a saliency map. Finally, the saliency map was segmented to detect salient regions and to locate object centers.
Fig. 2 Image edges and multiscale blobs

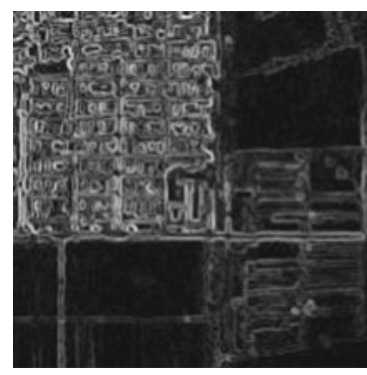

a) Edge (SOBEL)

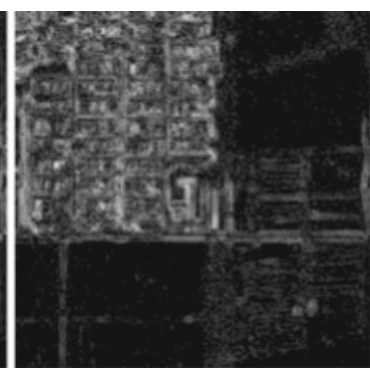

b) Single-scale Blob

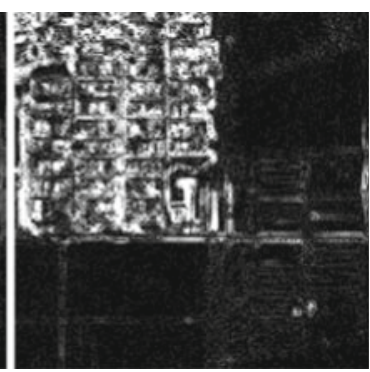

c) Multi-scale Blob 


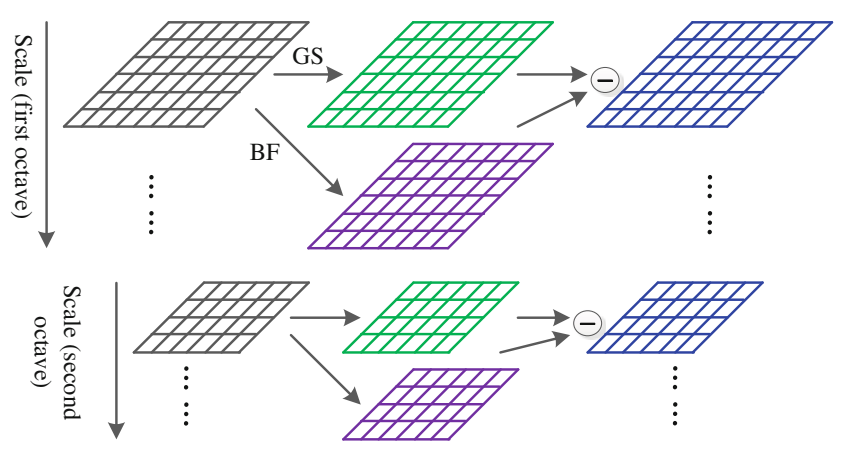

Fig. 3 Multi-scale blob detection

\section{Stepwise Algorithm}

\section{Multi-scale Blobs}

Multi-scale blobs were taken as the information source for saliency detection, rather than image edges in the existing methods (Rosin 2009; Hu et al. 2013). In images, edges just indicate intensity discontinuities, and appear on the boundaries of objects. While blobs consider diversity from its surround in scale, orientation, texture as well as intensity (Lowe 2004). Blobs are more universalistic (Lowe 2004) and provide more information of object saliency than image edges (Rosin 2009). Comparisons among edges, single-scale blobs and multi-scale blobs are shown in Fig. 2. Multi-scale blobs present more saliency information indicating ground objects than edges and blobs at a special scale, and suppress the background saliency.

A second variation was to look for blobs at multiple scales. Every object exists at a special scale in satellite images, and a single scale is not suitable to detect and describe saliency for all objects (Chalmond et al. 2006; Zhang et al. 2015). So multi-scale blobs were used to detect saliency map, as illustrated in Fig. 3.

To detect blob information from satellite images in multiple scales, we employed the multi-scale framework of SIFT features (Lowe 2004) and proposed a cascade smoothing and filtering approach. In the framework, we firstly operated Gaussian smoothing algorithm $G$ (Shapiro, 2001) and bilateral filter algorithm B (Yang et al. 2009) on every image $I$ in every scale in every octave, to produce a smoothed images $S$ (Eq. 1) and a filtered image $F$ (Eq. 2)respectively. Then we calculated a difference image $D$ between the smoothed image and the filtered image using Eq. 3, to indicate blob information of this scale.

$$
\begin{aligned}
& S\left(x, y, \sigma_{s}\right)=G\left(x, y, \sigma_{s}\right)^{*} I(x, y) \\
& F\left(x, y, \sigma_{s}, \sigma_{c}\right)=B\left(x, y, \sigma_{s}, \sigma_{c}\right) * I(x, y) \\
& D\left(x, y, \sigma_{s}\right)=\left|S\left(x, y, \sigma_{s}\right)-F\left(x, y, \sigma_{s}, \sigma_{c}\right)\right|
\end{aligned}
$$

where * is the convolution operation in $x$ and $y, \sigma_{s}$ is kernel standard deviation in coordinate space which is an adaptive parameter in the framework, $\sigma_{c}$ is kernel standard deviation to measure degree of changes in spectral space, and set to 50 for results reported in this paper.

After obtaining the difference image of every scale, we combined them to produce the final multi-scale blobs. Rather than find the local maxima and minima of difference images of multiple scales in the SIFT framework, we averaged the summation of all difference images to produce a local blob density $b_{d}$, using Eq. 4 . To incorporate distribution evenness $b_{e}$ of blob density in difference maps, we employed Eq. 5 to measure how evenly the blob were distributed in scale space. Finally, we used Eq. 6 to calculate the blob index $\boldsymbol{b}$ and generate a blob map. The blob map was normalized to [0255], where higher values indicate greater saliency. The blob density, the blob evenness and the blob index are demonstrated in Fig. 4. Combining the blob density and the blob evenness, blob map is more continuous and stable.

Fig. 4 The blob map

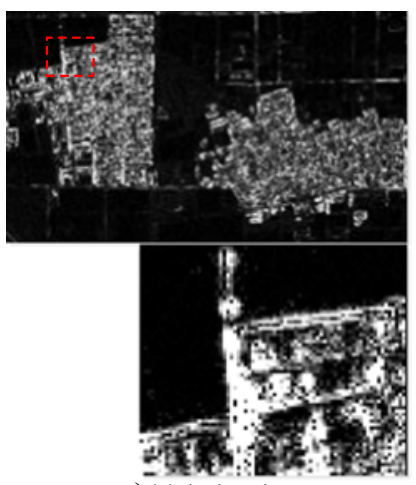

a) blob density

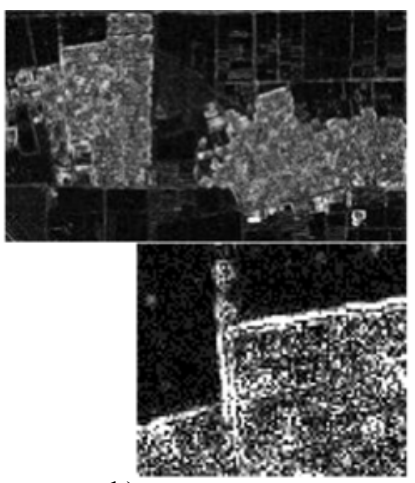

b) blob evenness

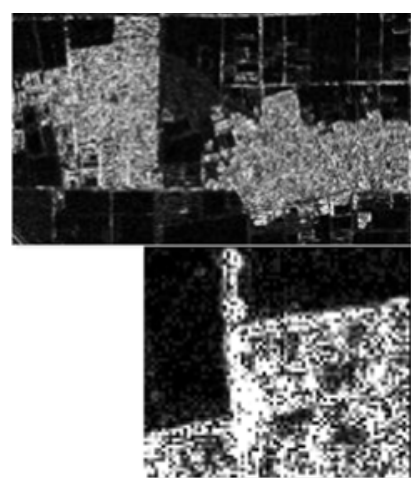

c) blob map 
Fig. 5 The summed saliency map and the minimum saliency map

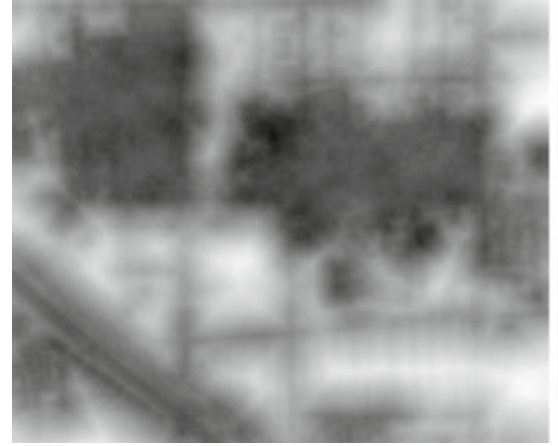

a) the summed saliency map

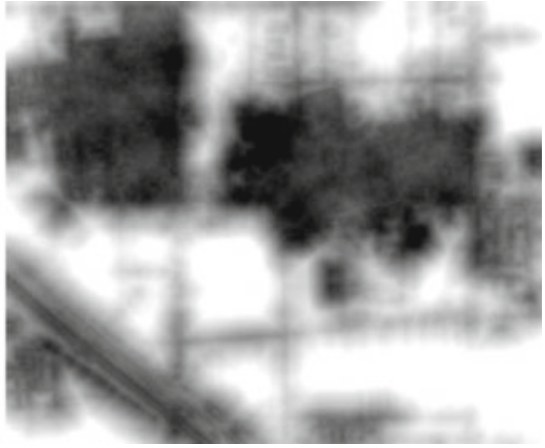

b) the minim saliency map
The scattered dark holes in bright regions are smaller in the blob map than that in the density map and the evenness map.

$$
\begin{aligned}
& b_{d}=\frac{1}{n} \sum_{n} d_{i} \\
& b_{e}=\left(\max \left(d_{i}\right)-\min \left(d_{i}\right)\right) / n \\
& b=b_{d} \times b_{e}
\end{aligned}
$$

where $n$ is the number of difference maps, $d_{i}$ is the value in the difference map $i$. Evenness $b_{e}$ ensures the blob index can capture salience changes in scale space. The blob index tends to be greater only if in regions with larger blob density and larger distribution evenness.

\section{Multi-level Distance Transform}

The detected blobs distribute discontinuously in images like honeycombs. A blurring was applied on the blob map for a smooth saliency maps. However, this would need choosing an appropriate kernel size for each image. To avoid requiring any parameters to be set, we used distance transform method to propagate the blob saliency. Since the standard distance transform method was defined for binary images rather than gray level images, a multi-level distance transform method was employed (Rosin 2009).

Firstly we used threshold decomposition to slice the blob map into multi-level binary maps. Supposed that the threshold value for decomposing the gray image $G(x, y)$ to generate the $i$-level binary image $B_{i}(x, y)$ is $T_{i}$. The $i$-level binary image can be produced using Eq. 7. As Rosin (2009) did, we chosen 64 gray thresholds from 0 to 255 with 4 for interval equably. This is $T_{i}=4 * i$.

$B_{i}(x, y)= \begin{cases}0, & G(x, y)>T_{i} \\ 255, & \text { otherwise }\end{cases}$

Then each binary map was processed separately to produce a distance transform map, using the standard distance transform method. Finally the set of distance transform map was combined to generate the salient map.

We conducted minimum operation (retrieving the minimum pixel gray value with the same position) on the set of distance transform maps to generate a smooth saliency maps $T$ in which lower values indicated greater saliency, instead of
Fig. 6 Salient regions and salient objects detection

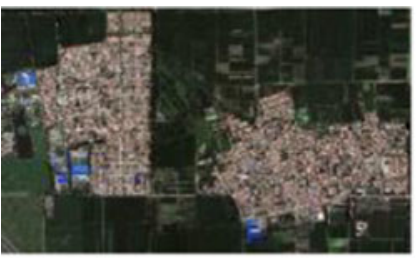

a1)

b1)

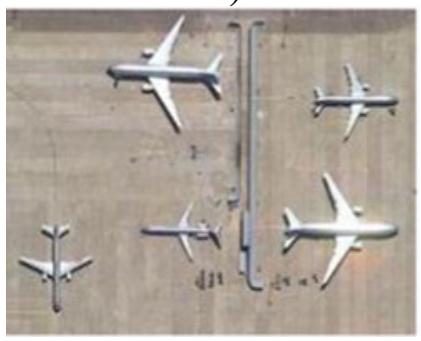

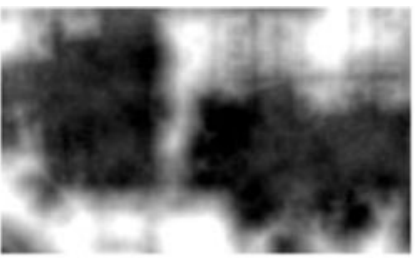

a2)

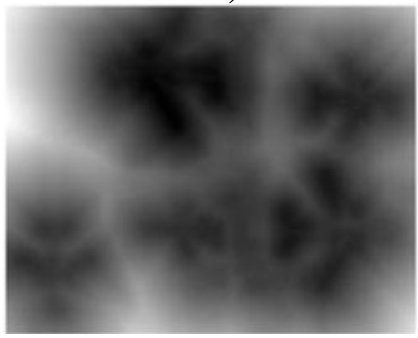

b2)

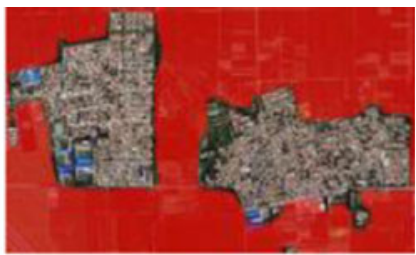

a3)

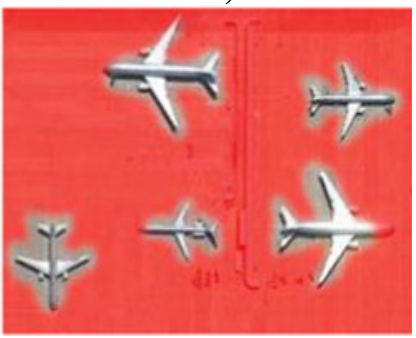

b3) 
Fig. 7 Detection of salient regions. The left column shows the images with the reference ground truths, the middle column presents the extracted salient regions by Rosin's method using $L$ channel of the experimental image, and the right column shows results of our method
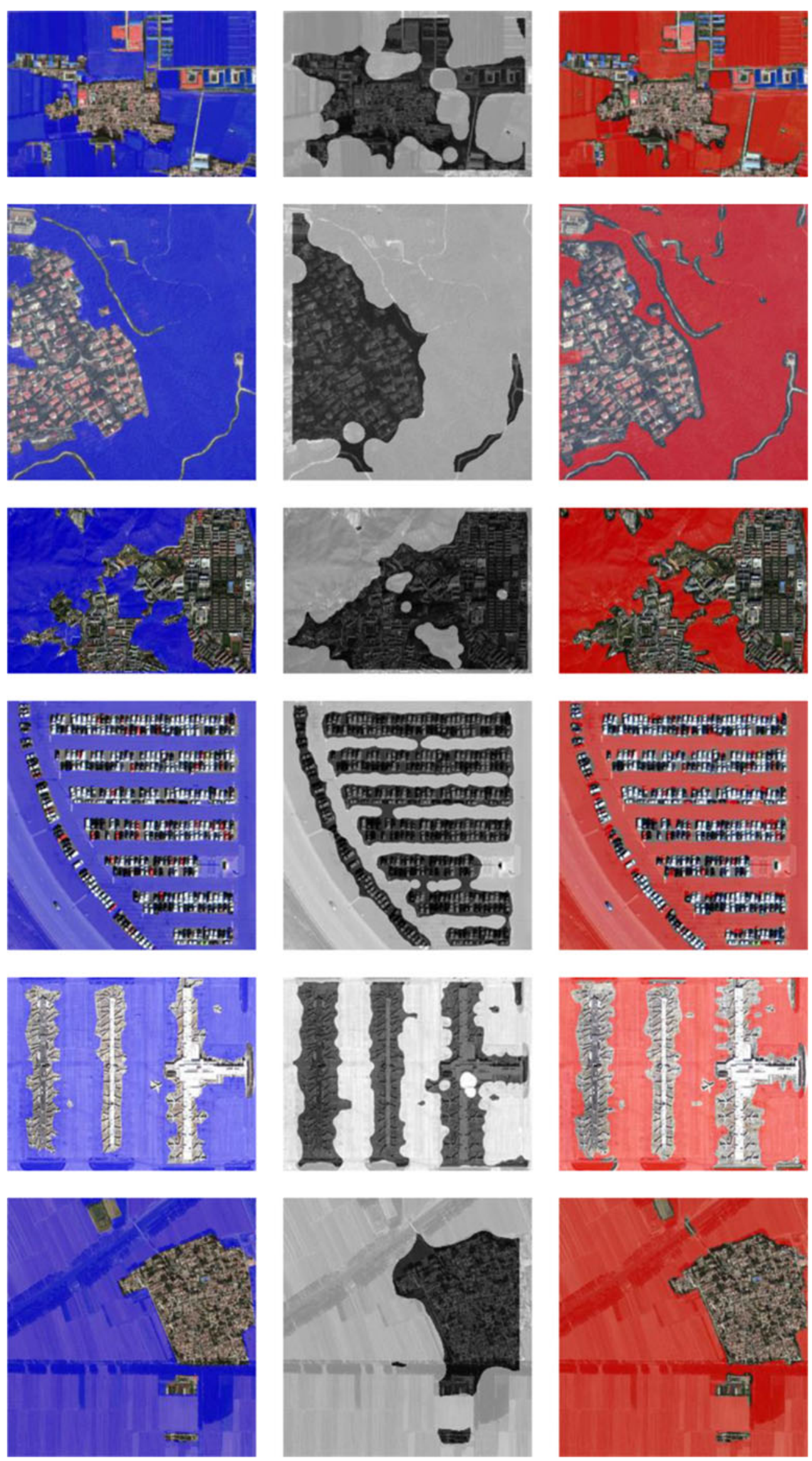
summation of the set of distance transform maps in Rosin (2009). The minimum map had a smaller variety than the summed map in local regions, which was important for salient region segmentation in next step. A comparison between the summed saliency map and the minimum saliency map is shown is Fig. 5.

\section{Salient Region/Object Segmentation}

To detect salient regions and objects, the saliency map $T$ was segmented into two classes, salient regions with saliency values lower than a certain threshold and background, using the Otsu method (Otsu 1975). The Otsu method is an adaptive global thresholding algorithm without any input parameters. Figure 6 presents the salient regions and salient objects after saliency map segmentation and edge smoothing.

\section{Experiments in Satellite Images}

The proposed approach is aimed at pointing potential regions of interest with a high detection ratio. It also can be designed to be followed by further recognition steps for special tasks, i.e. locating object centers (Hu et al. 2013; Wang et al. 2013). Two experiments (one for detecting salient regions, the other for locating object centers) were performed to evaluate the proposed method, using a number of satellite images with various resolutions and scene complexities.

\section{Salient Region Detection}

The first experiment was designed to extract salient regions, such as parking lots, residential areas, and airplane docks, in satellite images. All images were downloaded from Google Earth at their best resolutions for visual interpretation. Ground truths were combination of labelling results from various experts. Several experts first labelled the ground truths from the experimental satellite image. Then the labelled results were intersected into one result. Finally boundaries of the result were smoothed to produce the reference ground truths.

Some images and their detection results are presented in Fig. 7, compared with Rosin's method. Results showed that the two methods have detected the right salient regions. While our method has established better-defined and more compact boundaries for salient regions, closer to the reference ground truths.

We also assessed our method and Rosin's approach quantitatively, referring to the ground truths. Given the ground truths, performance for each image was defined in the precision-recall framework. The relative frequency of good and false decisions were calculated firstly. Then precision (Eq. 8) is defined as the ratio of true positive decisions $(T P)$ over all positive decisions $(T P+F P)$, while recall (Eq. 9) is the ratio of true positives over the number of expected good decisions (nP) (Chalmond et al. 2006). F-value (Eq. 10) is defined as harmonic combination of precision and recall for an overall performance measure, and $\boldsymbol{\alpha}$ is set to 0.5 .

precision $=T P /(T P+F P)$

recall $=T P / n P$

$F_{\alpha}=\frac{(1+\alpha) \times \text { precision } \times \text { recall }}{\alpha \times \text { precision }+ \text { recall }}$

Using F-value index, we evaluated 220 more satellite images representing different sensors, scene complexities, and land cover types. A comparison of $F$-value between Rosin's method and the proposed method is shown in Fig. 8.

The proposed method achieved a better performance with a mean $F$-value of 0.85 for the 220 images, than Rosin's method with a mean $F$-value of 0.70 . And the proposed method was more stable with a standard deviation $F$-value of 0.08 , compared with 0.12 for Rosin's method. This could be explained that Rosin's method only exploited edge information at a special scale, while our approach incorporated more blob information (density and evenness) of multiple scales. So the proposed method was more stable and could provide more homogeneous saliency values within regions (as presented in Fig. 4), and resulted in more precise delineation of region boundaries.

\section{Object Center Locating}

For some salient regions containing one object, we were able to locate their coordinate centers (object center) further. The point with the local maximum saliency values and within the local windows was chosen as the salient object center in $\mathrm{Hu}$ et al. 2013). It may be not very reasonable, since pixels with highest edge density and evenness of edge distribution mostly appear at object edges, not object centers.

We argued that the saliency value in local regions around object obey normal distribution, and pixels with saliency value in $[u-s, u+s]$ of the distribution are candidate object

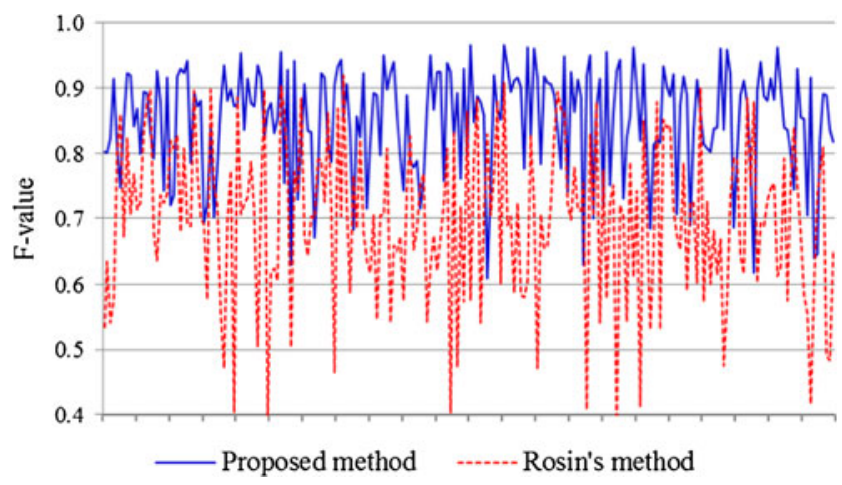

Fig. 8 F-value of Rosin's method and the proposed method on 220 satellite images 
Fig. 9 Center locating for ground objects. In d), red points represent centers of our method, green points represent centers of Hu's method, and blue points represent the reference centers

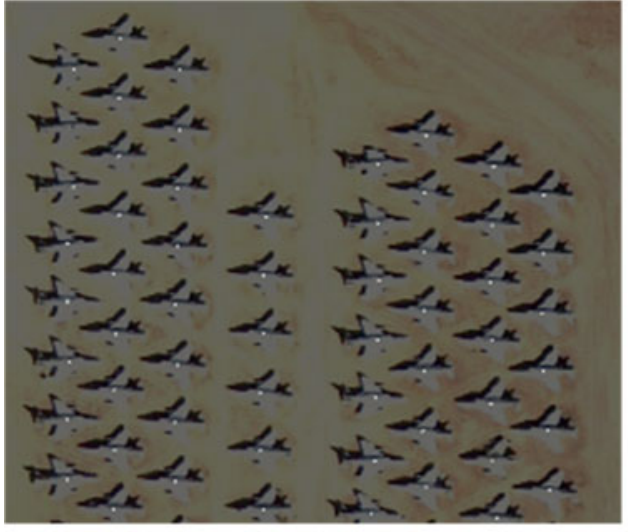

a) Reference object centers

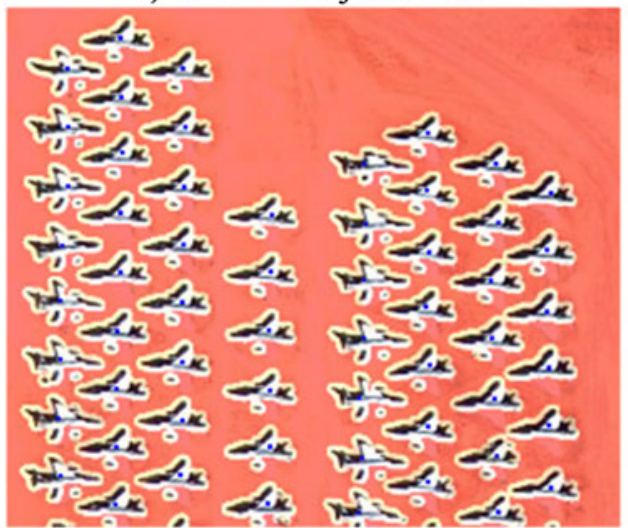

c) Center location of our method

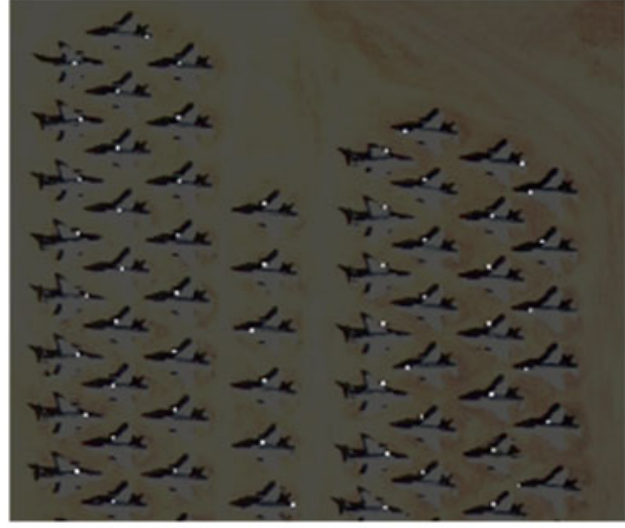

b) Center location of Hu's method

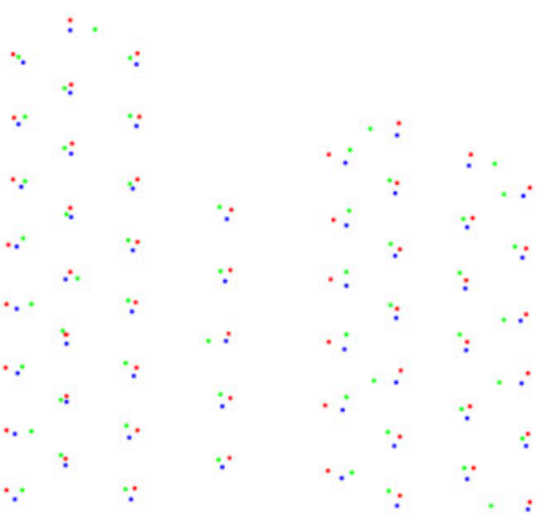

d) Comparison of located centers centers, where $u$ is the mean value and $s$ is standard deviation of saliency values in local regions. So in our method, pixels with the $68 \%$ highest saliency values in a local salient region were looked up firstly. Then object centers were selected as the weighted sum of the locations of the found pixels.

Compared with Hu's method, we evaluated the proposed method, referring plane centers delineated on the geometrical center of planes by experts, as shown in Fig. 9. In Hu's method (Hu et al. 2013), saliency values based on edge information guided object centers to the strongest edge points with highest saliency index in local regions (wings of airplanes, not the actual plane center), as shown in Fig. 9b). While the proposed

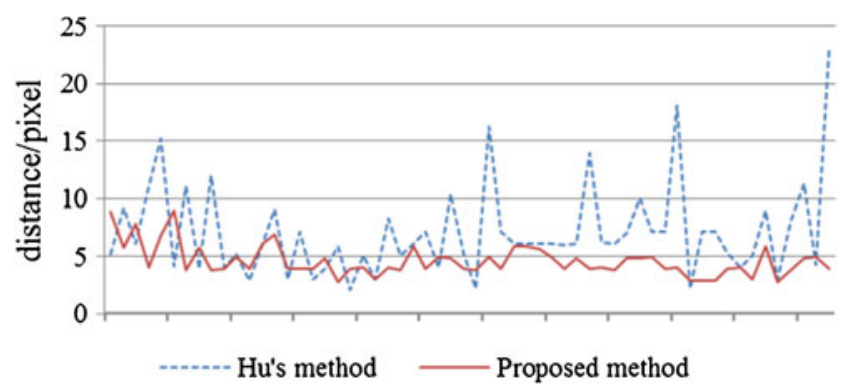

Fig. 10 Assessments of object center location method located object centers on bodies of planes, as shown in Fig. 9c), which outperformed Hu's method.

We noted that there was a systematic error in our detected airplane centers, resulting from shadows of airplanes in satellite images. Deflecting from the airplane locations, the shadows provide false blob information for the saliency map. And the salient regions as well as object centers translate along the shadow direction, as shown in Fig. 9.

Measuring the distance from a detected center to its reference center, we evaluated the performance for locating plane centers of the proposed method, as presented in Fig. 10. Our method has substantially outperformed Hu's method, with a mean value of center deflection of 4.62 pixels and a standard deviation of center deflection of 1.35 pixels, compared with 7.11 pixels and 4.06 pixels for Hu's method, upon all the 58 planes. Our method produced more stable results, and had a higher position accuracy of centers.

\section{Conclusion and discussion}

This paper presented an automatic and effective approach based on multi-scale blobs for salient region detection and object center location in satellite images. 
Incorporating blob information of multiple scales, the approach outperformed available methods in detecting saliency regions and locating object centers. Results of salient region detection on 220 images showed the proposed method is more precise and more stable than Rosin's method, producing well-defined region boundaries. Further experiment on plane center location also illustrated promising results in precision and stability of center positions.

However, as partially shown in the second experiment, the image-based method can hardly handle knowledge-based ground objects (such as airplane shadows), resulting in deflection of detection. Incorporating high-level knowledge (such as shadow removal technique) is a further work. On the other hand, we discarded color information of images in our method. Color or spectrum features are fundamental for analysis and understanding of satellite images. Further works will take it into account.

Acknowledgments This work was supported in part by China Postdoctoral Science Foundation under Grant 2015M570138, in part by the National Natural Science Foundation of China under Grant 41301488 and 41301438 , in part by the National High Technology Research and Development Program of China (863 Program) under Grant 2013AA12A401-2, in part by the National Major Program on High Resolution Earth Observation System under Grant 03-Y30B06-9001-13/15-01.

Conflict of interest The authors report no conflict of interest. The authors are responsible for the content and writing of the paper.

\section{References}

Chalmond, B., Francesconi, B., \& Herbin, S. (2006). Using hidden scale for salient object detection[J]. Image Processing, IEEE Transactions on, 15(9), 2644-2656.

Chen, H. Z., Jing, N., Wang, J., Chen, Y. G., \& Chen, L. (2014). A novel saliency detection method for lunar remote sensing images[J]. Geoscience and Remote Sensing Letters, IEEE, 11(1), 24-28.

Cui, X., Tian, Y., \& Ma, L. (2014). Top-Down Visual Saliency Detection in Optical Satellite Images Based on Local Adaptive Regression Kernel[J]. Journal of Multimedia, 9(1), 173-180.
Harel J, Koch C, Perona P. Graph-based visual saliency[C]//Advances in neural information processing systems. 2006: 545-552.

Hu, X., Shen, J., Shan, J., \& Pan, L. (2013). Local edge distributions for detection of salient structure textures and objects[J]. Geoscience and Remote Sensing Letters, IEEE, 10(3), 466-470.

Itti, L., Koch, C., \& Niebur, E. (1998). A Model of SaliencyBased Visual Attention for Rapid Scene Analysis[J]. IEEE Transactions on Pattern Analysis and Machine Intelligence, 20(11), 1254-1259.

Jäger, M., \& Hellwich, O. (2005). Saliency and salient region detection in SAR polarimetry[C]. International Geoscience And Remote Sensing Symposium, 4, 2791.

Klein, D. A., \& Frintrop, S. (2011). Center-surround divergence of feature statistics for salient object detection[C] Computer Vision (ICCV), 2011 I.E. International Conference on. IEEE, 2011: 2214-2219.

Li, Z., \& Itti, L. (2011). Saliency and gist features for target detection in satellite images[J]. Image Processing, IEEE Transactions on, 20(7), 2017-2029.

Li, W., \& Pan, C. (2011). Saliency-based automatic target detection in remote sensing images[M] Advanced Research on Computer Science and Information Engineering. Springer Berlin Heidelberg, 2011: 327-333

Lowe, D. G. (2004). Distinctive image features from scale-invariant keypoints[J]. International Journal of Computer Vision, 60(2), 91110.

Otsu, N. (1975). A threshold selection method from gray-level histograms[J]. Automatica, 11(285), 23-27.

Rosin, P. L. (2009). A simple method for detecting salient regions[J]. Pattern Recognition, 42(11), 2363-2371.

Shapiro, L. G., \& Stockman, G. C. (2001). Computer vision. Prentence Hall, 137-150.

Wang, X., Lv, Q., Wang, B., \& Zhang, L. (2013). Airport detection in remote sensing images: a method based on saliency map $[\mathrm{J}]$. Cognitive Neurodynamics, 7(2), 143-154.

Xu G, Huo H, Fang T, \& Li D. (2007). Extracting salient object from remote sensing image based on guidance of visual attention[C] International Symposium on Multispectral Image Processing and Pattern Recognition. International Society for Optics and Photonics, 2007: $67902 \mathrm{~W}$

Yang, Q., Tan, K. H., \& Ahuja, N. (2009). Real-time O(1) bilateral filtering[C] Computer Vision and Pattern Recognition, 2009. CVPR 2009. IEEE Conference on. IEEE, 557-564.

Zhang, L., Qiu, B., Yu, X., \& Xu, J. (2015). Multi-scale hybrid saliency analysis for region of interest detection in very high resolution remote sensing images[J]. Image and Vision Computing, 35, 1-13. 